\title{
Role of Turbulences in WDM-Polarization Interleaving Scheme based Inter-Satellite Communication System
}

\author{
Sushank Chaudhary \\ InterNetworks Research Lab \\ School of Computing \\ Universiti Utara Malaysia, \\ Malaysia
}

\author{
Saurabh Sharma \\ ECE Department \\ Institute of Science \& \\ Technology, Klawad
}

\author{
Parveen \\ ECE Department \\ Institute of Science \& \\ Technology, Klawad
}

\begin{abstract}
Inter-Satellite communication is the revolutionary technology used to transmit the signals between the satellites. This work is focused to carry out the investigation of turbulences in Inter-Satellite communication system by incorporating WDMPI interleaving scheme. A 6 x 20 Gbps channels are transported over Inter-Satellite link having span of $1000 \mathrm{~km}$ to realize the total transmission of $120 \mathrm{Gbps}$. The role of transmitter pointing errors and receiving pointing errors in the OWC link is investigated and results are reported in terms of SNR, total received power and eye diagrams.
\end{abstract}

\section{General Terms}

Inter-Satellite Communication, Transmitter Pointing Error, Receiving Pointing Error, Polarization Interleaving, WDM.

\section{Keywords}

Inter-Satellite Communication, Transmitter Pointing Error, Receiving Pointing Error, Polarization Interleaving, WDM.

\section{INTRODUCTION}

Laser communication is currently fit to send information at rate up to several Gbps and at a separation of a large number of kilometers. This has opened up a thought to embrace an optical remote communication system for between satellite connection generally performed utilizing microwave [1]. An inter-satellite optical wireless communication (Is-OWC) system offers enormous bandwidth and high data speed which makes them an attractive mode of meeting the constantly rising demand of broadband services [2-3]. The higher directivity of the optical beam allows higher data/power efficiency, i.e. more Mbps for each watt of power. The main driving force behind the introduction of photonics in space communication is the reduction in mass, volume and power of the payload. Regardless of the above advantages of Is-OWC link, the satellite communication systems are the most difficult classification of optical wireless link, as a result of the long separations that the optical beam propagates through vacuum or the air [4]. Optical connections that work in a vacuum (between satellite and space communications) are at any rate a huge number of $\mathrm{kms}$ long. Regardless of the fact that the vacuum is thought to be perfect (lossless), the related path losses that expand with the square of the connection separation are enormous. Subsequently, a key attention in satellite communications is the received optical power and a few strategies have been proposed to relieve the transmission related losses. Optical amplification is an engaging hopeful [5-9], particularly in earth-to-satellite uplinks. Subsequently, the utilization of Free Space optics for ground communications systems are also investigated by researchers [10-13]. The concept of space based optical reaches up to several Tbps which bring the concept to maturity and the requirement of inter-satellite communication paid towards the inter-satellite optical wireless link which is characterized by receiving signal power at receiver side [9] and is given by

$$
\mathrm{P}_{\mathrm{R}}=\mathrm{P}_{\mathrm{T}} \eta_{T} \eta_{R}\left(\frac{\lambda}{4 \pi Z}\right)^{2} G_{T} G_{R} L_{T} L_{R}
$$

Where $\mathrm{P}_{\mathrm{T}}$ is the transmitter optical power, $\eta_{T}$ and $\eta_{R}$ are the optical efficiency of the transmitter and receiver respectively, $\lambda$ is the wavelength, $Z$ is the distance between the transmitter and the receiver, $G_{T}$ is the transmitter telescope gain, $G_{R}$ is the receiver telescope gain, $L_{T}$ and $L_{R}$ are the transmitter and receiver pointing loss factor respectively. The free space path loss is described by the terms in the parentheses in the above equation. Longer links typically require more correct and advanced recovery, pointing and tracking system to keep up an operational optical wireless link, since the smallest error is amplified by the link distance. So, the imperative requirement of Is-OWC system is that the sender and receiver antenna must be in line of sight, even the small beam divergence can cause signal loss. The approximation transmitting pointing loss factor is given by:

$L_{T}=\exp \left(-G_{T} \theta_{T}^{2}\right)$

where $\theta_{T}$ is transmitter azimuth pointing error angle, and the approximate receiver pointing error is given by the following equation:

$L_{R}=\exp \left(-G_{T} \theta_{T}^{2}\right)$

Where $\theta_{R}$ is the receiver azimuth pointing error. In the recent years there has been growing interest to develop technologies that can fulfill the bandwidth concern of bandwidth starved applications. One such technique for accessing the huge bandwidth capacity in an optical link is wavelength division multiplexing (WDM). This technology puts data from different sources together in a single optical link with each signal carried at the same time on different wavelengths [1415]. By utilizing WDM technology the connection limit of any optical system could be expanded up to several tera-hertz. The non-linear impacts have a tendency to show themselves when optical channel spacing is low and the data rates are high, as in the case of DWDM systems [16-18]. In our proposed system, we used a polarization interleaving technique (PI) to reduce such non-linearity. In this PI technique, the state of polarization of the optical signal in successive channels is kept orthogonal to each other. The remainder of the paper is formed as follows: Section II portrays the system description; Section III depicts the results and discussion emulated by Section IV which depicts the conclusion. 


\section{SYSTEM DESCRIPTION}

In our Is-OWC by incorporating WDM-PI technique, strategy as shown in Fig 1 designed in OptiSystem ${ }^{\mathrm{TM}}$ software, 6 channels, each one having 20 Gbps Non Return-Zero (NRZ) information, is carried and modulated over light sources of 0 $\mathrm{dBm}$ operating at $1545 \mathrm{~nm}$ with the dispersing of $0.5 \mathrm{~nm}$ through a Mach Zehnder modulator (MZM). The six channels, i.e. Channel 1, Channel 2, Channel 3, Channel 4, Channel 5 and Channel 6 are divided into odd and even channels and then multiplexed separately. The output of the two multiplexers is then nourished to polarization controller (PC), which changes the azimuth parameter of the even and odd channels so that the contiguous signals are orthogonal to one another. These polarized signals are again multiplexed together and transmit to the receiver through an optical wireless channel (OWC). The OWC is comprised of a transmitter and receiver antenna having an aperture diameter of $20 \mathrm{~cm}$ and $30 \mathrm{~cm}$ respectively. The antennas are assumed to be ideal and their optical efficiency is kept equivalent to 1 . Furthermore, the losses due to mispointing of the transmitter and receiver are investigated at a distance of $1000 \mathrm{Km}$, i.e. the receiver and transmitter pointing error angle is varied from $1 \mu \mathrm{rad}$ to $5 \mu \mathrm{rad}$. The receiver side of the Is-OWC system contains a polarization splitter (PS) which divides the received signal according to their state of polarization (SOP) i.e. into even and odd signals. The output of PS is then demultiplexed and detected by an avalanche photodiode (APD) followed by the Bessel low pass filter with a cutoff frequency of $15 \mathrm{GHz}$.

\section{RESULTS AND DISCUSSION}

In this section, the results of the proposed simulated setup of WDM-PI based Is-OWC system is presented and discussed. Fig. 2 illustrates the effect of transmitter pointing error angle on the total power received and SNR for channels 1, 3 and 6 at a distance of $1000 \mathrm{Km}$ for the different operating wavelength of OWC channel, i.e. $850 \mathrm{~nm}$ and $1550 \mathrm{~nm}$ in terms of SNR and total received power at the photo detector. As the transmitting pointing error reaches $5 \mu \mathrm{rad}$, The channel 1, channel 3 and channel 6 shows an improvement of $-20 \mathrm{dBm}$ in total power and $20 \mathrm{~dB}$ in SNR is noticed when the OWC channel is operated at $1550 \mathrm{~nm}$ as compared to $850 \mathrm{~nm}$ shown in Fig. 2 (a), (b), (c), (d), (e) \& (f). Similarly the eye diagrams for channels 2 , channel 4 and channel 5 after the transmission of $1000 \mathrm{~km}$ is reported in the Fig 3. It has depicted from the fig 3 that for the transmitting pointing error of $3 \mu \mathrm{rad}$, the eye diagrams are distorted with high bit error rate when the OWC channel is operated at $850 \mathrm{~nm}$ as compared to $1550 \mathrm{~nm}$.

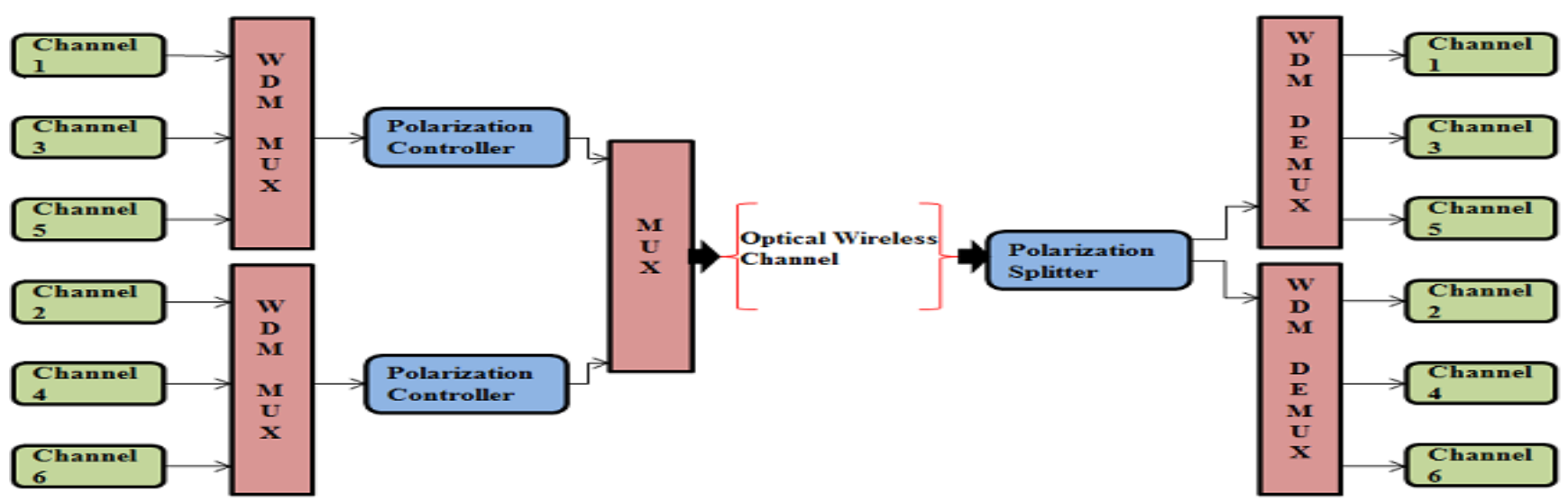

Fig. 1 Proposed Hybrid WDM-Polarization Interleaving Is-OWC System 
Total Power Vs Transmitter Pointing Error

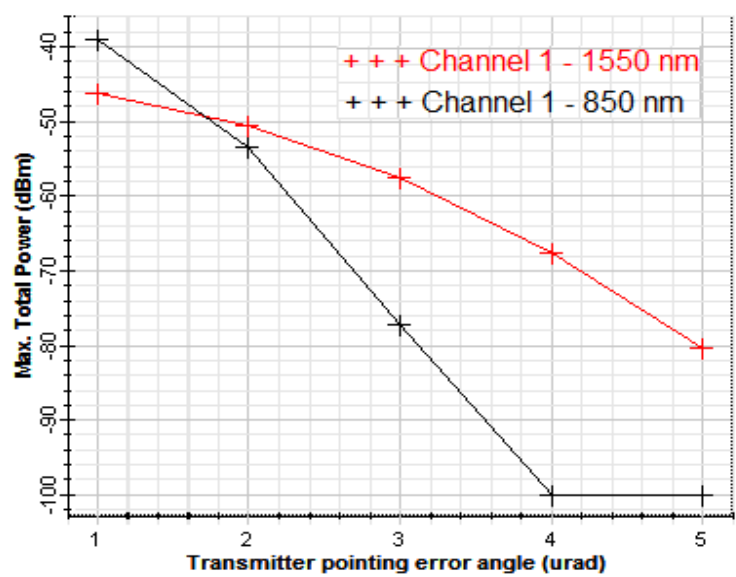

(a)

Total Power Vs Transmitter Pointing Error

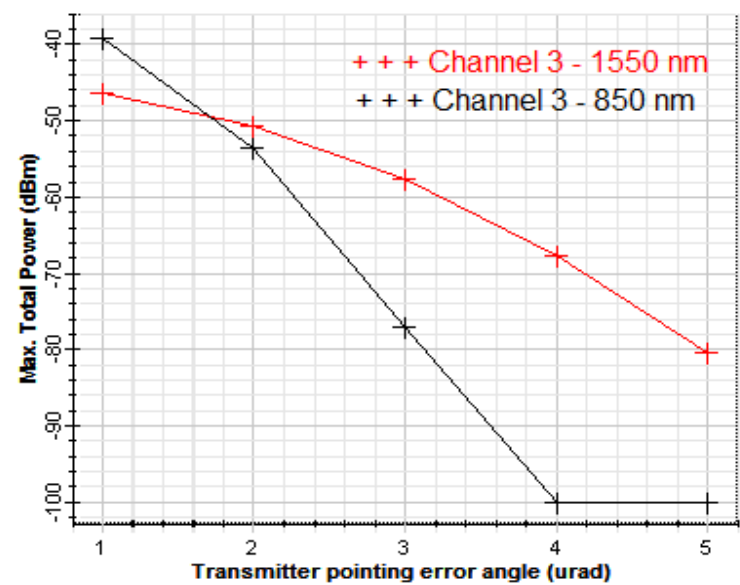

(c)

Total Power Vs Transmitter Pointing Error

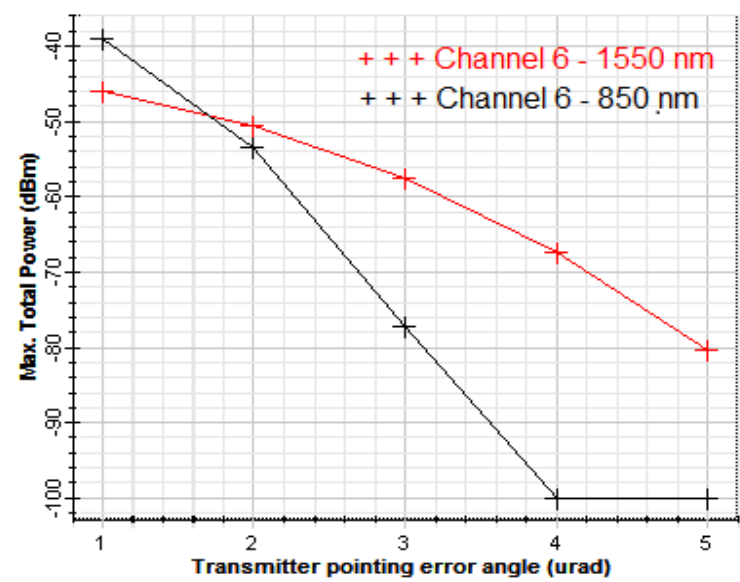

(e)
SNR Vs Transmitter Pointing Eror

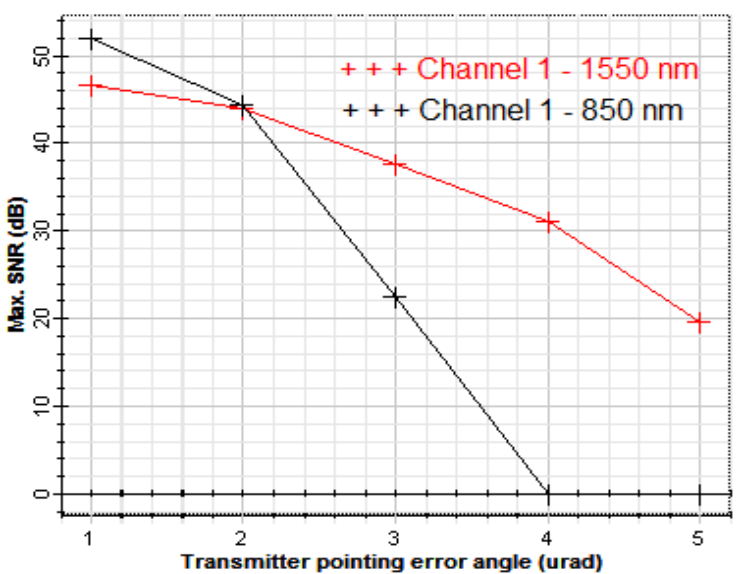

(b)

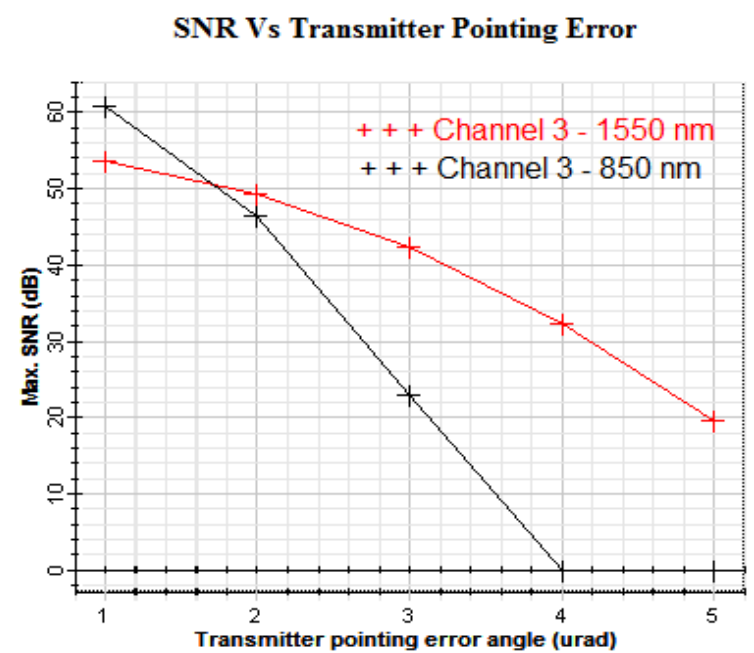

(d)

SNR Vs Transmitter Pointing Error

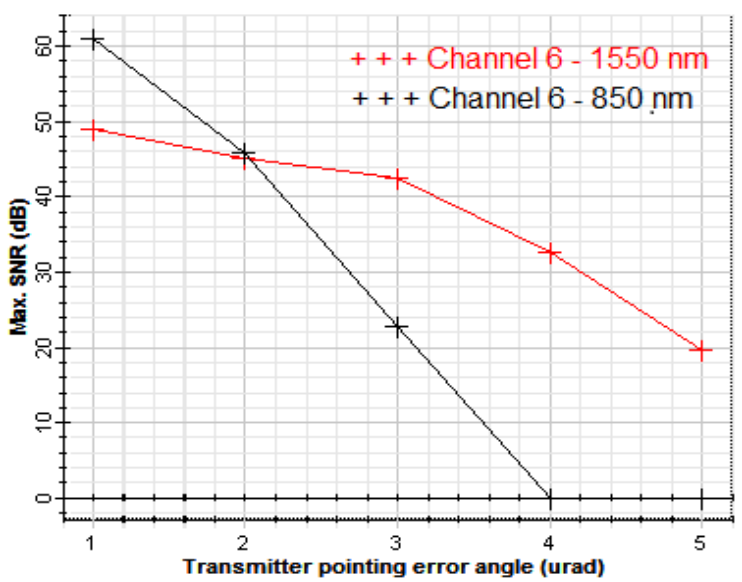

(f)

Fig 2: Total Received Power and SNR Vs Transmitter Pointing Error for (a \& b) Channel 1 (c \& d) Channel 3 (e \& f) Channel 6 

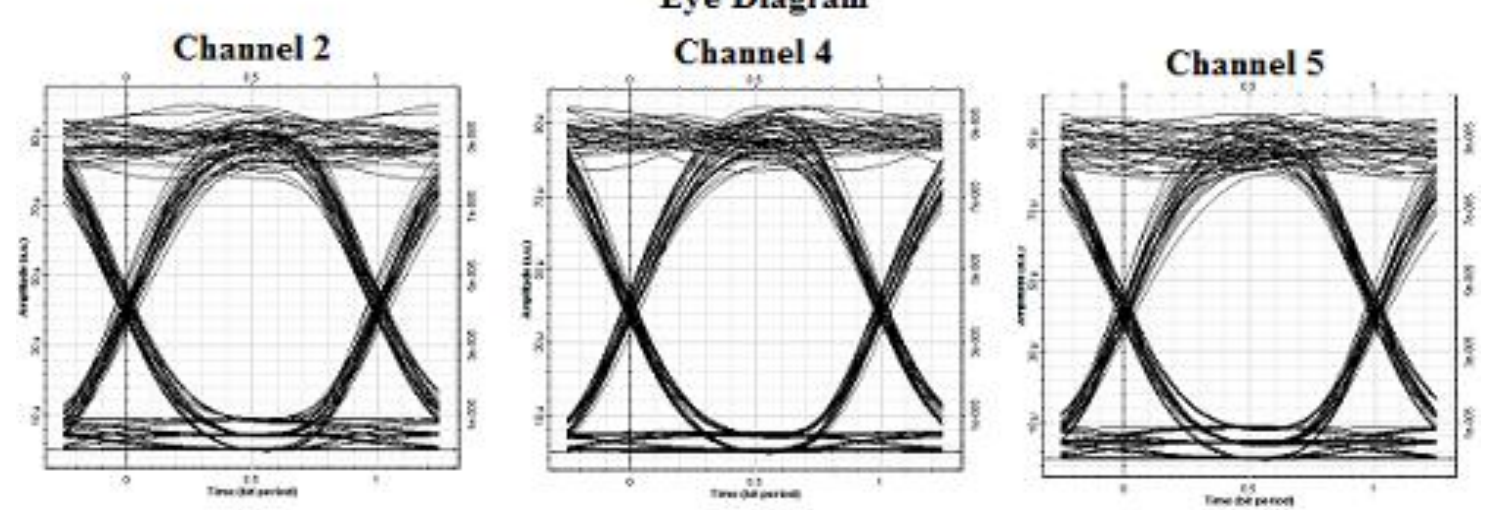

At $1550 \mathrm{~nm}$
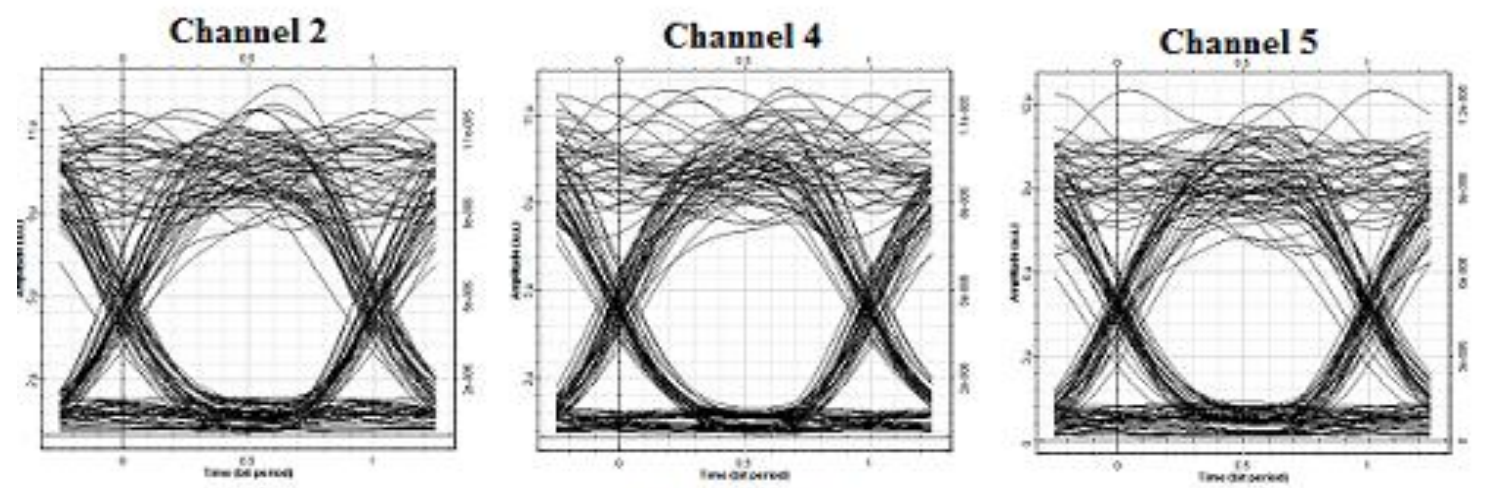

At $850 \mathrm{~nm}$

Fig 3: Eye Diagram at transmitter pointing error of $3 \mu \mathrm{rad}$

Total Power Vs Receiver Pointing Error

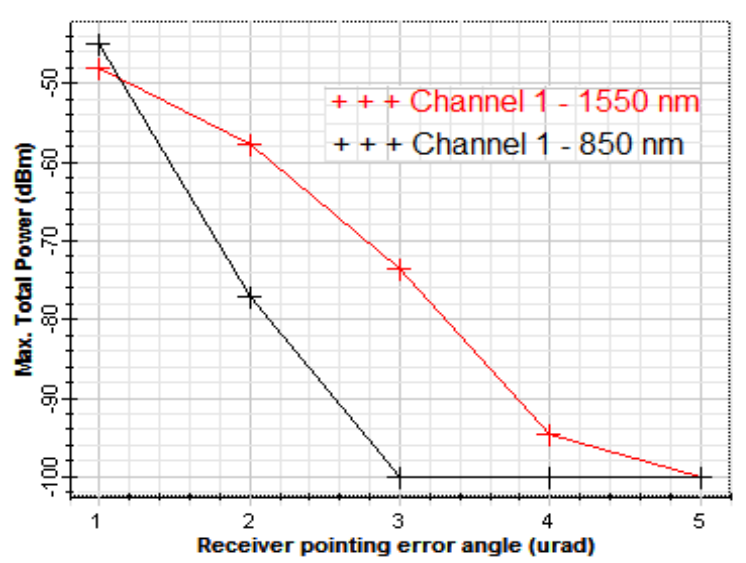

(a)
SNR Vs Receiver Pointing Error

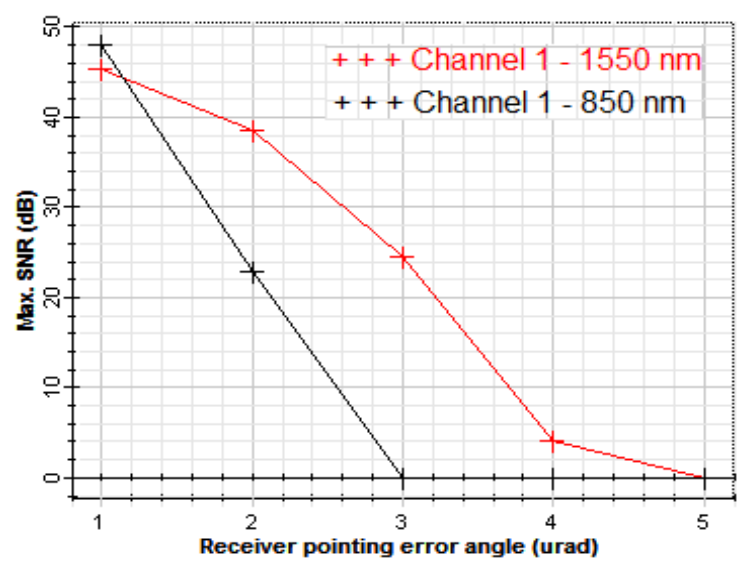

(b) 
Total Power Vs Receiver Pointing Error

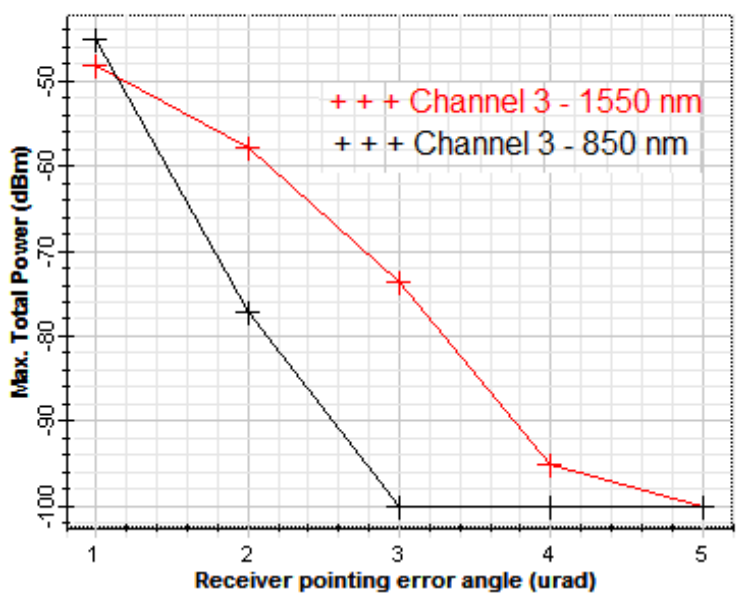

(c)

Total Power Vs Receiver Pointing Error

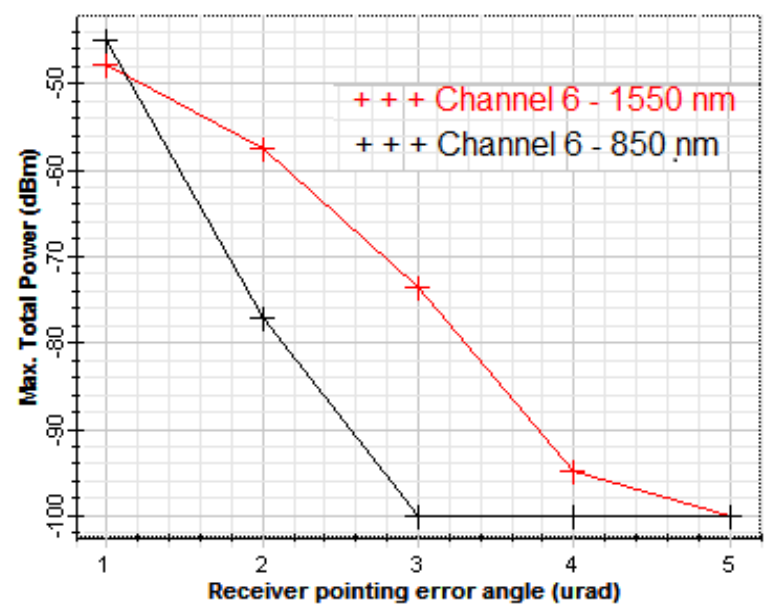

(e)
SNR Vs Receiver Pointing Error

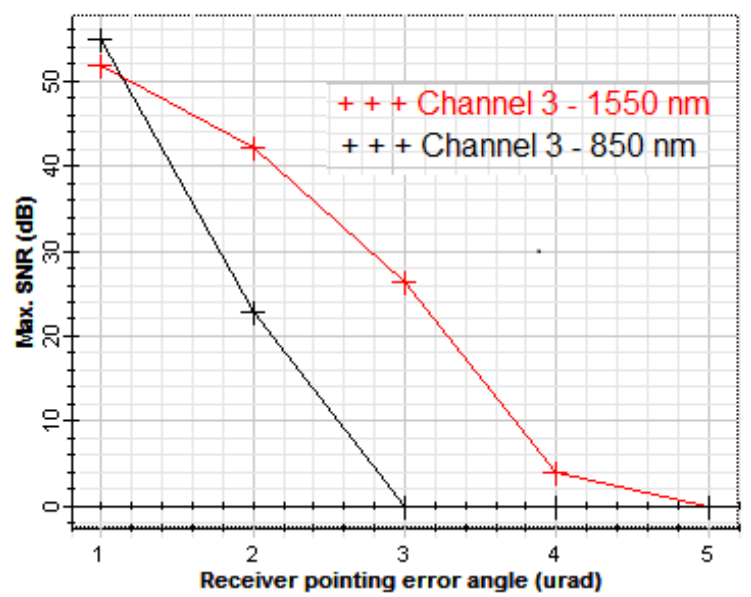

(d)

SNR Vs Receiver Pointing Error

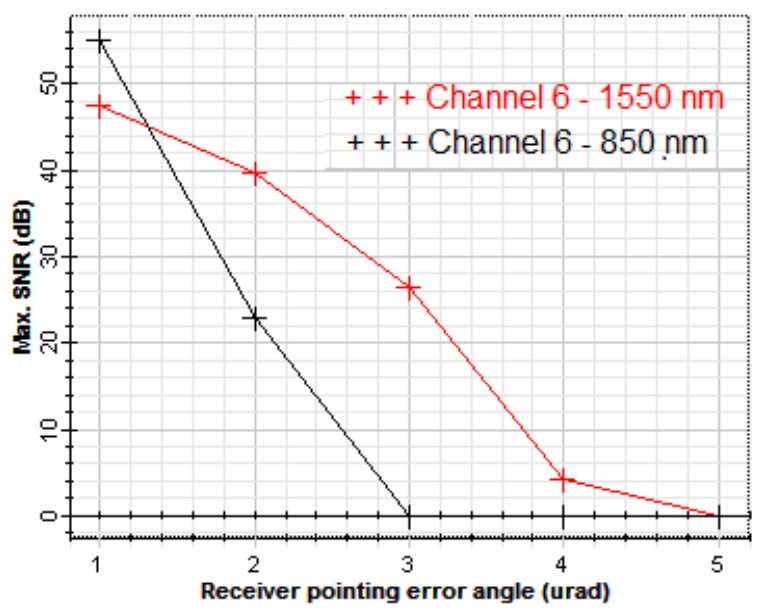

(f)

Fig 4: Total Received Power and SNR Vs Receiver Pointing Error for (a \& b) Channel 1 (c \& d) Channel 3 (e \& f) Channel 6 

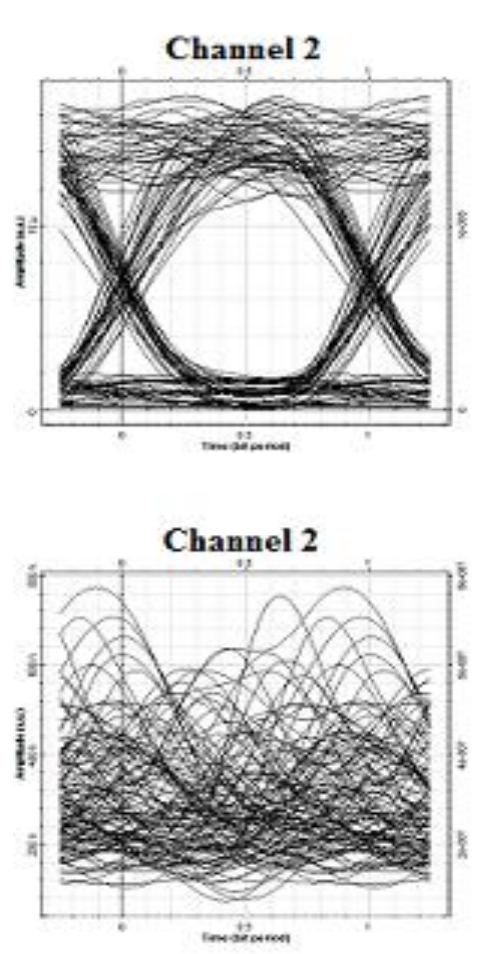

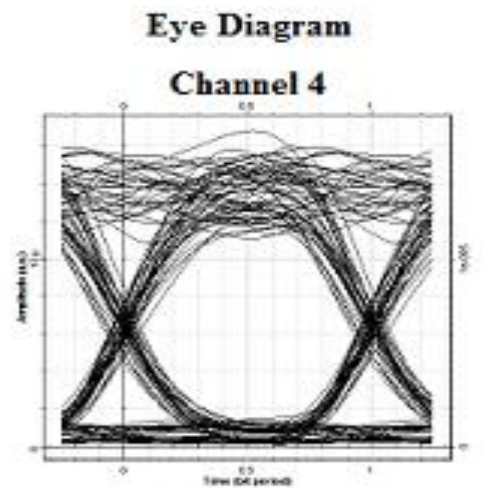

At $1550 \mathrm{~nm}$

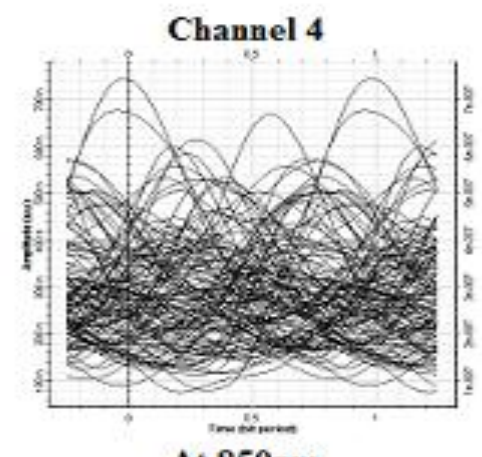

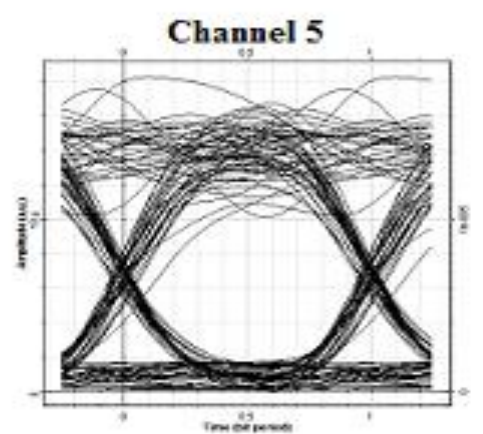

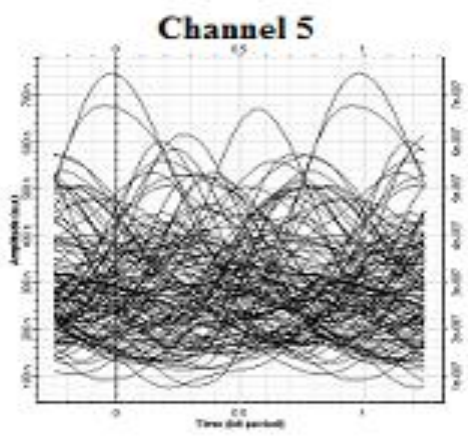

At $850 \mathrm{~nm}$

Fig 5: Eye Diagram at receiver pointing error of $3 \mu \mathrm{rad}$

Similarly, the effect of receiver pointing error is also analyzed in the proposed system which is shown in Fig 4 and 5. It has been noticed that the effect of a receiving pointing error is more distorted as compared to the transmitting pointing error. As the receiving pointing error reaches $5 \mu \mathrm{rad}$, both OWC channels operated at $850 \mathrm{~nm} \& 1550 \mathrm{~nm}$ for all the channels has degraded SNR and total received power. However, the OWC channel operated at $1550 \mathrm{~nm}$ has acceptable SNR and total received power when the receiving pointing error is fixed to $3 \mu \mathrm{rad}$ for all channels which is also illustrated in Fig 5 .

\section{CONCLUSION}

In this work, we have designed a high speed hybrid WDM-PIIs-OWC system which is capable of transmitting the 120 Gbps over inter-satellite optical link of $1000 \mathrm{Km}$ between two satellites under turbulences of transmitter and receiver pointing error angle. Furthermore, we compared the operating wavelength of OWC channel. From our results, it is concluded that the proposed WDM-PI scheme is beneficial for designing the Inter-Satellite communication system by considering the transmitting and receiving pointing errors. It is presumed that under transmitting and receiving pointing errors, the OWC link performs better operating at $1550 \mathrm{~nm}$ with acceptable SNR, BER and total received power.

\section{ACKNOWLEDGEMENT}

The author would like to thank Mr. Sanjay Gupta COO of GigaSoft India for providing the valuable resources in order to complete this research work.

\section{REFRENCES}

[1] A. H. Hashim, "Modeling and performance study of intersatellite optical wireless communication system", International Conference on Photonics (ICP), IEEE, pp.1-4, 2010.
[2] M. A. Krainak, "Inter-satellite communications optoelectronics research at the Goddard Space Flight Center", Aerospace and Electo-system Magazine, IEEE, vol. 7, pp.44-47, 1992.

[3] Sushank Chaudhary and Angela Amphawan "The Role and Challenges of Free-space Optical Systems" Journal of Optical Communications. Volume 0, Issue 0, ISSN (Online) 2191-6322, ISSN (Print) 01734911, DOI: $10.1515 /$ joc-2014-0004

[4] Naresh Kumar, "2.50 Gbit/s optical wireless communication system using PPM modulation schemes in HAP-to-satellite links", Optik - International Journal for Light and Electron Optics, Volume 125, Issue 14, July 2014, Pages 3401-3404, ISSN 0030-4026.

[5] IL Pe'er, N Naftali, A Yogev, "High power, solar pumped, Nd:YAG, laser amplifier for free space laser communication”, Proc SPIE 3139, 194-204 (1997).

[6] E Rochat, R Dändliker, K Haroud, RH Czichy, U Roth, D Costantini, R Holzner, "Fiber amplifiers for coherent space communication", IEEE J Sel Topics Quantum Electron 7(1), 64-81 (2001).

[7] A. Polishuk, S. Arnon, "Optimization of a laser satellite communication system with an optical preamplifier", J. Optical Society of America. Vol. 21, No. 7, pp 13071315, July 2004.

[8] JW Dawson, MJ Messerly, RJ Beach, MYSEA Stappaerts, AK Sridharan, PH Pax, JE Heebner, CW Siders, CPJ Barty, Analysis of the scalability of diffraction-limited fiber lasers and amplifiers to high average power. OSA Opt Express 16(17), 13240-13266 (2008). 
[9] S. Arnon, "Performance of a laser satellite network with an optical preamplifier", J. Optical Society of America. Vol. 22, No. 4, pp 708-715, April 2005.

[10] Sushank Chaudhary, Preety Bansal and Gurdeep Singh "Implementation of FSO Network under the Impact of Atmospheric Turbulences" International Journal of Computer Applications 75(1):34-38, August 2013.

[11] Vishal Sharma and Sushank Chaudhary "Implementation of Hybrid OFDM-FSO Transmission System" International Journal of Computer Applications 58(8):37-40, November 2012.

[12] Sushank Chaudhary, Angela Amphawan, Kashif Nisar, "Realization of free space optics with OFDM under atmospheric turbulence", Optik - International Journal for Light and Electron Optics, Available online 8 July 2014, ISSN 0030-4026

[13] Vishal Sharma, Sushank, "High speed CO-OFDM-FSO transmission system", Optik - International Journal for Light and Electron Optics, Volume 125, Issue 6, March 2014, Pages 1761-1763, ISSN 0030-4026

[14] Borella, M., Jue, J., Banerjee, D., Ramamurthy, B. and Mukherjee, B., "Optical components for WDM lightwave networks", Proceedings of the IEEE, Vol. 85 No. 8, August 1997, pp. 1274-1307.

[15] Cho P.S, Gtigoryan V.S, Godin Y.A, Salamon A, Achiam Y, "Transmission of $25 \mathrm{~Gb} / \mathrm{s}$ RZ-DPSK Signals with $25 \mathrm{GHz}$ channel spacing over $1000 \mathrm{~km}$ SMF-28 fiber," Photon. Technol. Lett. Vol.15, No.3, pp. 473-475, March 2003.

[16] A. R. Chraplyvy, "Limitations on lightwave communications imposed by optical fiber nonlinearities," J. Lightw. Technol., vol. 8, pp. 1548-1557, 1990.

[17] Sushank Chaudhary, Preety Bansal and Manisha Lumb "Effect of Beam Divergence on WDM-FSO Transmission System" International Journal of Computer Applications 93(1):28-32, May 2014

[18] Ramandeep Kaur and Sushank Chaudhary "Simulative Investigation of Laser Line-width and Channel Spacing for Realization of DWDM Systems under the Impact of Four Wave Mixing" Journal of Optical Communications. Volume 35, Issue 2, Pages 157-165, ISSN (Online) 2191-6322, ISSN (Print) 01734911, DOI: 10.1515/joc-2013-0152, March 2014. 